\title{
Obstructive sleep apnea and insight into mechanisms of sympathetic overactivity
}

\author{
François Abboud and Ravinder Kumar
}

Department of Internal Medicine and Department of Molecular Physiology and Biophysics, University of lowa Carver College of Medicine, Iowa City, lowa, USA.

\begin{abstract}
Nearly two decades ago, we evaluated ten patients with obstructive sleep apnea (OSA). We determined that alarming nocturnal oscillations in arterial pressure and sympathetic nerve activity (SNA) were caused by regulatory coupling and neural interactions among SNA, apnea, and ventilation. Patients with OSA exhibited high levels of SNA when awake, during normal ventilation, and during normoxia, which contributed to hypertension and organ damage. Additionally, we achieved a beneficial and potentially lifesaving reduction in SNA through the application of continuous positive airway pressure (CPAP), which remains a primary therapeutic approach for patients with OSA. With these results in hindsight, we herein discuss three concepts with functional and therapeutic relevance to the integrative neurobiology of autonomic cardiovascular control and to the mechanisms involved in excessive sympathoexcitation in OSA.
\end{abstract}

\section{Neural reflex interactions determine optimal autonomic cardiovascular response}

An integrated autonomic response to combined stress signals is essential for optimal adjustment. For example, in the cardiovascular system, circulatory responses are typically mediated through activation of the sympathetic and parasympathetic branches of the autonomic system and serve to maintain blood flow and oxygen delivery to vital organs $(1,2)$.

The oxygen-conserving reflex. Responses to obstructive sleep apnea (OSA) are triggered by hypoxia and apnea. During hypoxia, chemoreceptor activation promotes hyperventilation to enhance oxygen delivery to blood, which is followed by sympathetically mediated vasoconstriction to redistribute oxygenated blood flow to vital organs $(1,2)$ and parasympathetically activated bradycardia to reduce myocardial oxygen demand (3). Within each respiratory cycle, the cardiovascular response is maximal during expiration and suppressed during inspiration (4); however, this inhibitory coupling of respiratory afferent nerves with the chemoreceptor-mediated excitation of both sympathetic and parasympathetic efferents is dysfunctional during apnea $(1,3)$. Apnea unbridles an intense

Conflict of interest: The authors have declared that no conflict of interest exists.

Citation for this article: J Clin Invest. 2014; 124(4):1454-1457. doi:10.1172/JCI70420. cardiovascular response in order to maintain oxygen delivery, reduce cardiac oxygen demand, and promote survival. This is an oxygen-conserving reflex, similar to the evolutionarily conserved diving reflex in seals and ducks, that occurs in humans during facial immersion and apnea (5-7). Unfortunately, this physiologic response to hypoxia becomes pathological when the enhanced sympathoexcitation is sustained over years, as is the case in OSA.

Baroreceptor-chemoreceptor interaction. Several sensory signals converge during stress, resulting in an integrated reflex response. We have reported several examples of occlusive and facilitatory central interactions in humans (Figure 1). For example, there is a central interaction between heat sensation in the extremities and arterial baroreceptor afferents that prevents baroreflex vasoconstriction locally, preserving the need for heat dissipation while maintaining arterial pressure (8). Another important interaction is the coordination between increased arterial pressure and baroreceptor activity, which overrides chemoreceptor reflex to prevent hyperventilation and reflex vasoconstriction $(9,10)$. In hypertensive humans, hypoxia and apneaassociated chemoreceptor responses are exaggerated, enhancing both sympathetic nerve activity (SNA) and parasympathetic bradycardia $(3,11,12)$. The enhanced chemoreceptor reflex associated with hypertension is likely attributable, at least in part, to simultaneous baroreceptor reflex suppression $(12,13)$. Studies by our group revealed that baroreceptor activation suppresses chemoreceptor-mediated SNA increases $(9,11,12)$; subsequently, Zucker et al. revealed that carotid sinus nerve stimulation provides a survival advantage in a canine heart failure model (14). Together, these results provided rationale for recent clinical trials to evaluate electrical stimulation of carotid sinus nerves as an antihypertensive therapy in patients with drug-resistant hypertension (15). Preliminary results are promising and indicate a dual benefit of sympathoinhibition and parasympathetic activation for patients in heart failure. We believe that the beneficial SNA suppression is the result of both arterial baroreflex-mediated sympathoinhibition and suppression of chemoreceptor-mediated sympathoexcitation (11). Elegant studies by Schreihofer and colleagues have provided electrophysiological evidence that the central interaction between baroreceptor and chemoreceptor signals and the central respiratory modulation of SNA during acute hypoxia occurs in neurons of the caudal ventrolateral medulla (CVLM) $(16,17)$.

The carotid body in bypertension. The finding that excessive SNA associates with chemoreceptor overactivity and hypertension led to multiple studies on the drivers of glomus cell activation in response to hypoxia and acidosis. Identified molecular determinates include the electron transport chain, reactive oxygen, $\mathrm{NO}$, and $\mathrm{K}^{+}$ channels (reviewed in ref. 18). We determined that overexpression of the acidicsensing ion channels (ASICs) and two-pore domain $\mathrm{K}^{+}$channels enhanced $\mathrm{pH}$ sensitivity in glomus cells in young spontaneously hypertensive rats (SHRs) prior to hypertension onset (4). Additionally, we found that carotid body resection had an antihypertensive effect when performed in young prehypertensive SHRs. In fact, resection reduced systolic pressure rise over a fivemonth period in SHRs (19). Similar results were observed and published contemporaneously by Paton and colleagues (20). Cur- 
A

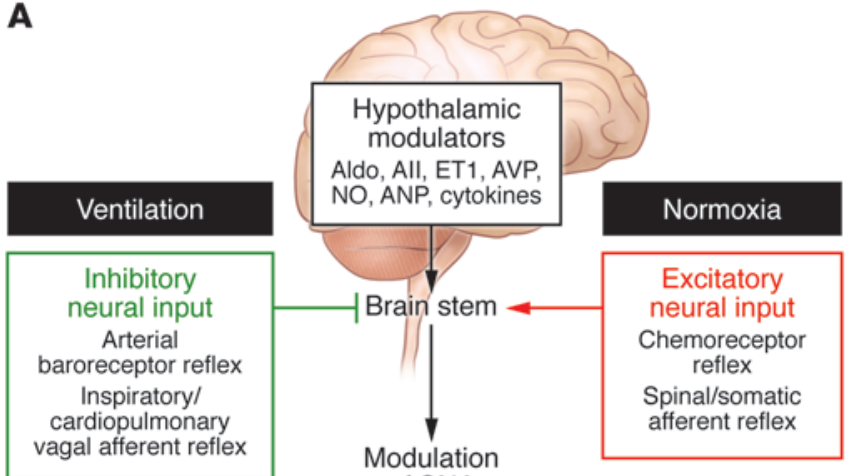

of SNA

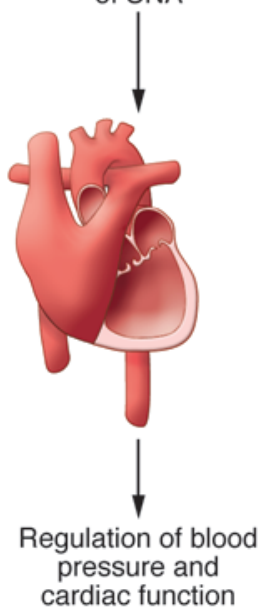

B

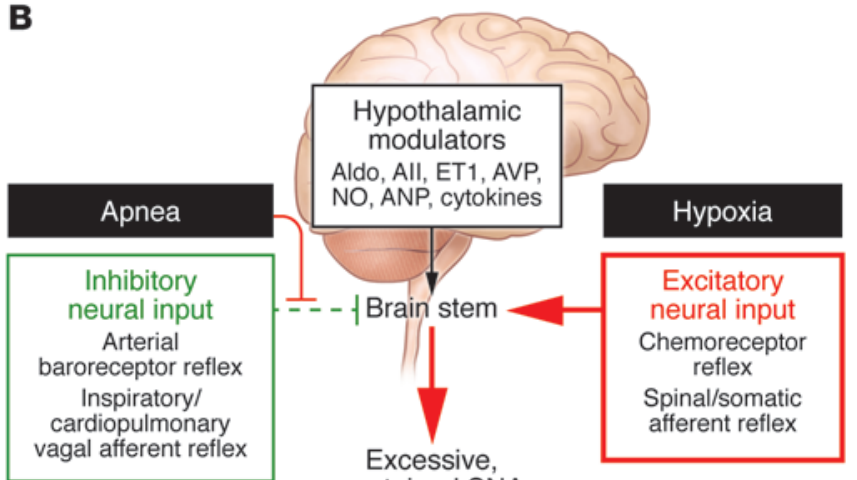

sustained SNA

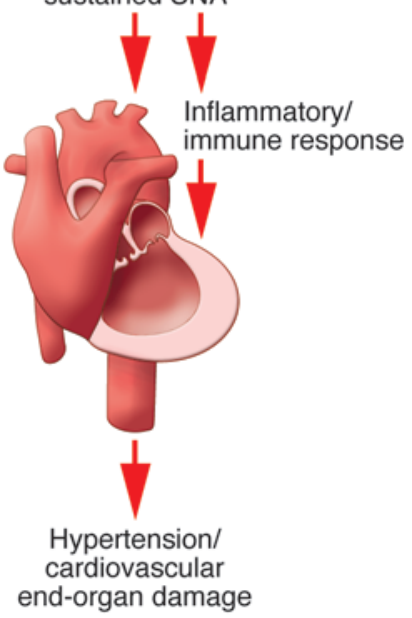

Figure 1

Enhancement of SNA promotes cardiovascular disease. (A) Under normal conditions, hypothalamic modulators, including aldosterone (Aldo), angiotensin II (All), endothelin 1 (ET1), arginine vasopressin (AVP), NO, atrial natriuretic peptide (ANP), and cytokines, influence SNA. In healthy individuals, SNA is promoted by excitatory neural input (red) in response to peripheral stress. Simultaneously, peripheral responses (green), such as the arterial baroreceptor reflex and the cardiopulmonary and other vagal afferent reflexes, buffer the increase in SNA and maintain homeostasis. (B) Patients with OSA exhibit sustained excessive SNA, due to a pathological increase of excitatory neural input (red) and prevention and/or decrease of the protective inhibitory signals (green). Sustained SNA promotes proinflammatory immune responses and, ultimately, cardiovascular disease-associated end-organ damage.

rently, carotid body resection along with carotid sinus nerve stimulation, vagal nerve stimulation, and renal sympathetic denervation are under consideration as options for treating drug-resistant hypertension and heart failure $(21,22)$.

\section{Neuroplasticity and neuroimmune mechanisms sustain OSA- associated sympathoexcitation and organ damage}

The inappropriate sustained SNA increase in OSA patients likely contributes to hypertension, organ damage, and mortality; however, it is unclear how excessive SNA develops in these patients. Several factors, including obesity and increased carotid body chemoreceptor sensitivity due to intermittent hypoxia, have been considered. Obesity could mechanically obstruct the airway and increase SNA through leptin, insulin, angiotensin, and cytokine actions; however, many OSA patients are not obese (23). Carotid body hypersensitivity as a result of intermittent hypoxia has been confirmed in animal models of OSA. In fact, plasticity of the carotid body glomus cells with long-term sensory facilitation and sensitization have been reported $(18,24)$ and associated with ROS and NOX2-dependent accumulation of HIF1 and the transcriptional coactivator CREBbinding protein (25).

Central neuroplasticity. A provocative possibility for OSA-associated SNA dysfunction is that excessive activation of CNS nuclei induces a process of neuroplasticity that increases the excitatory drive to the rostral ventrolateral medulla (RVLM) and sustains a high sympathetic tone independently of the peripheral sensory signals (Figure 1 and ref. 26).
We previously reported such an excessive central excitatory drive of renal SNA in aging ten-year-old beagles, whose baseline renal nerve activity was drastically higher than predicted based on total deafferentation of the inhibitory aortic, carotid, and vagal afferents (27). Recently, Johnson and colleagues reported that angiotensin II sensitization induces a molecular neurogenic substrate in central neurons that promotes hypertension (28). Furthermore, the authors revealed that after a delay period of sensitization, prohypertensive molecules were pronounced in critical CNS sites, thus provoking an increase in SNA. Intriguingly, induction of prohypertensive molecules may also result from various environmental and psychogenic stresses, including episodic periods of hypoxia, as observed in OSA. Molecular sensitization may be sustained by positive feedback, which 
could explain the sustained hypertensive effect associated with chronic intermittent hypoxia as reported by Cunningham (29).

Neuroimmune mechanisms. The immune system contributes to cardiovascular diseases, including arrhythmias, hypertension, atherosclerosis, and heart failure (30). Tissue injury promotes release of damageassociated molecular pattern molecules (DAMPs) that in turn activate receptors on innate immune cells or resident inflammatory cells in various organs, such as microglia in the CNS (31). Receptor activation triggers cytokine release into the circulation or at specific brain nuclei, which can induce neuronal sympathoexcitation and enhance the homing of inflammatory cells to vulnerable end-organs. In the CNS, the inflammatory response results in sympathoexcitatory effects that induce positive feedback, and signals associated with increased SNA induce mobilization of hematopoietic stem and progenitor cells and their proliferation (32).

During the past decade, several studies have demonstrated an interaction between the autonomic system and the immune system. Harrison's group convincingly demonstrated a critical role for $\mathrm{T}$ lymphocytes in angiotensin II-induced sympathoexcitation and hypertension (33). We found that the innate immune system of genetically hypertensive rats is abnormally proinflammatory due to activation of autonomic receptors on immune cells, which enhances their inflammatory response to TLR activation $(34,35)$. The link between the autonomic system and the immune system may promote the marked accentuation of end-organ damage and increased mortality ascribed to excessive sympathoexcitation in myocardial infarction, hypertension, and heart failure. In fact, vagal nerve stimulation has an antiinflammatory effect (36), which may account for the dramatically prolonged survival documented in animal models of heart failure (37). While it is clear that OSA promotes a proinflammatory state, it remains to be seen whether antiinflammatory treatments can reduce and/or reverse the OSA-associated sustained sympathetic drive and delay or prevent organ damage (31).

\section{Could improvement of a simple device affect cardiovascular disease?}

OSA is an aggregate of dysfunctions, but characteristically, it is a chronic sleep disorder associated with incessantly recur- ring periods of apnea as a result of pharyngeal collapse.

Understanding the events that lead to pharyngeal collapse will be important for preventing this initial trigger of OSA onset. During sleep, inappropriate suppression of reflexive neural activation of pharyngeal dilator muscles could account for intermittent or complete pharyngeal collapse (38). Hypoxia and hypercapnia during an apneic episode incite a vigorous ventilatory effort and disrupt sleep to restore the pharyngeal neuromuscular drive and open the airway.

We clearly demonstrated that continuous positive airway pressure (CPAP) therapy promotes a beneficial reduction in blood pressure and SNA during sleep (1). Examination of CPAP therapy over a period of one to six months and its effect on various end points strongly supports its use (39). Unfortunately, despite modifications that have been made over two decades, many patients do not tolerate the CPAP facial mask, which maintains positive airway pressure. Considering the prevalence of OSA and the enormous cost of associated cardiovascular risks, further technological improvement of the pressure device to achieve uniform acceptance and exploration of new approaches to restore neuropharyngeal tone (40) could greatly affect health care outcomes.

\section{Conclusions and reflections}

With the benefit of hindsight, we lament, as many have, the decline in physicianscientists and the paucity of clinical investigation into physiologic mechanisms in healthy humans and in disease states, while an avalanche of drug-based trials make up the bulk of translational research.

We find the neurobiology of the autonomic nervous system a fascinating integrative process of connecting and converging sensory signals. In fact, the biology of brain function is quintessentially integrative, and President Obama has put an emphasis on the Brain Research through Advancing Innovative Neurotechnologies (BRAIN) initiative to usher in a decade of "connectomics."

Substantial advances have been made and will continue to define molecular substrates that determine central neuronal plasticity and influence chemosensitivity of glomus cells. Importantly, more work will need to be done to determine how diverse and heterogeneous environmental stressors, including intermittent hypoxia, induce sensitization of specific CNS sites in the hypothalamus and brain stem to provoke pathological and fatal excesses of SNA and hypertension. As opposed to the shortsighted assumption that the sympathetic nervous system primarily functions as a buffer for circulatory collapse, it is now recognized that the sympathetic nervous system fatally accelerates pathological proinflammatory immune processes.

\section{Acknowledgments}

F. Abboud recognizes the major contribution to the OSA study by Virend Somers, back when the author had the good fortune of recruiting him to Iowa as a senior postdoctoral fellow from Oxford. Somers, currently at the Mayo Clinic, has become a leading world authority on OSA. R. Kumar is a fellow in Cardiovascular Medicine. The work was supported by NIH grant HL-014388-42.

Address correspondence to: François Abboud, Department of Internal Medicine and Department of Molecular Physiology and Biophysics, University of Iowa Carver College of Medicine, 616 MRC, Iowa City, Iowa 52242-1009, USA. Phone: 319.335.7708; Fax: 319.335.6969; E-mail: francois-abboud@uiowa.edu.

1. Somers VK, Dyken ME, Clary MP, Abboud FM. Sympathetic neural mechanisms in obstructive sleep apnea. J Clin Invest. 1995;96(4):1897-1904.

2. Abboud FM, Thames MD. Interaction of cardiovascular reflexes in circulatory control. In: Shepherd J, Abboud F, eds. Handbook of Physiology: Peripheral Circulation and Organ Blood Flow. Bethesda, Maryland, USA: American Physiological Society; 1984:675-753.

3. Somers VK, Dyken ME, Mark AL, Abboud FM. Parasympathetic hyperresponsiveness and bradyarrhythmias during apnea in hypertension. Clin Auto Res. 1992;2(3):171-176.

4. Tan ZY, et al. Chemoreceptor hypersensitivity, sympathetic excitation, and overexpression of ASIC and TASK channels before the onset of hypertension in SHR. Circ Res. 2010;106(3):536-545.

5. Heistad DD, Abboud FM, Eckstein JW. Vasoconstrictor response to simulated diving in man. J Appl Physiol. 1968;25(5):542-549.

6. Irving L, Scholander PF, Grinnell SW. The regulation of arterial blood pressure in the seal during diving. Am J Physiol. 1942;135:557-566.

7. Folkow B, Nilsson NJ, Yonce LR. Effects of "diving" on cardiac output in ducks. Acta Physiol Scand. 1967;70(3):347-361.

8. Heistad DD, Abboud FM, Mark AL, Schmid PG. Interaction of thermal and baroreceptor reflexes in man. J Appl Physiol. 1973;35(5):581-586.

9. Somers VK, Mark AL, Abboud FM. Interaction of baroreceptor and chemoreceptor reflex control of sympathetic nerve activity in normal humans. J Clin Invest. 1991;87(6):1953-1957.

10. Heistad DD, Abboud FM, Mark AL, Schmid PG. Effect of baroreceptor activity on ventilatory response to chemoreceptor stimulation. J Appl Physiol. 1975;39(3):411-416.

11. Heistad DD, Abboud FM, Mark AL, Schmid PG. Interaction of baroreceptor and chemoreceptor reflexes. Modulation of the chemoreceptor reflex by 
changes in baroreceptor activity. J Clin Invest. 1974; 53(5):1226-1236

12. Somers VK, Mark AL, Abboud FM. Potentiation of sympathetic nerve responses to hypoxia in borderline hypertensive subjects. Hypertension. 1988; P2(6 pt 2):608-612.

13. Schultz HD, Li YL, Ding Y. Arterial chemoreceptors and sympathetic nerve activity: implications for hypertension and heart failure. Hypertension. 2007;50(1):6-13.

14. Zucker IH, et al. Chronic baroreceptor activation enhances survival in dogs with pacing-induced heart failure. Hypertension. 2007;50(5):904-910.

15. Wustmann K, et al. Effects of chronic baroreceptor stimulation on the autonomic cardiovascular regulation in patients with drug-resistant arterial hypertension. Hypertension. 2009;54(3):530-536.

16. Mandel DA, Schreihofer AM. Modulation of the sympathetic response to acute hypoxia by the caudal ventrolateral medulla in rats. $J$ Physiol. 2009;587:(pt 2):461-475

17. Silva AQ, Schreihofer AM. Altered sympathetic reflexes and vascular reactivity in rats after exposure to chronic intermittent hypoxia. J Physiol. 2011;589(pt 6):1463-1476.

18. Kumar P, Prabhakar NR. Peripheral chemoreceptors: function and plasticity of the carotid body. Comp Physiol. 2012;2(1):141-219.

19. Whiteis CA, Post CE, Morgan DA, Rahmouni K, Chapleau MW, Abboud FM. Peripheral chemoreceptors contribute significantly to hypertension in spontaneously hypertensive rats (SHR). FASEB J. 2012;26:703.15.

20. Abdala AP, et al. Hypertension is critically dependent on the carotid body input in the spontaneously hypertensive rat.J Physiol. 2012;590(pt 17):4269-4277.
21. Hauptman PJ, Mann DL. The vagus nerve and autonomic imbalance in heart failure: past, present, and future. Heart Fail Rev. 2011;16(2):97-99.

22. Simplicity HTN-2 Investigators, et al. Renal sympathetic denervation in patients with treatmentresistant hypertension (The Simplicity HTN-2 Trial): a randomized controlled trial. Lancet. 2010; 376(9756):1903-1909.

23. Gami AS, et al. Obstructive sleep apnea, obesity, and the risk of incident atrial fibrillation. $\mathrm{J} \mathrm{Am} \mathrm{Coll}$ Cardiol. 2007;49(5):565-571.

24. Prahakar NR, Peng YJ, Kumar GK, Pawar A. Altered carotid body function by intermittent hypoxia in neonates and adults: Relevance to recurrent apneas. Respir Physiol Neurobiol. 2007;157(1):148-153.

25. Peng YJ, et al. Heterozygous HIF-1-alpha deficiency impairs carotid body-mediates systemic responses and reactive oxygen species generation in mice exposed to intermittent hypoxia. J Physiol. 2006; 577(pt 2):705-716.

26. Guyenet PG. The sympathetic control of blood pressure. Nat Rev Neurosci. 2006;7(5):335-346.

27. Hajduczok G, Chapleau MW, Johnson SL, Abboud FM. Increase in sympathetic activity with age. I. Role of impairment of arterial baroreflexes. Am J Physiol Heart Circ Physiol. 1991;260(29):H1113-H1120.

28. Xue B, Zhang Z, Roncari CF, Guo F, Johnson AK. Aldosterone acting through the central nervous system sensitizes angiotensin II-induced hypertension. Hypertension. 2012;60(4):1023-1030.

29. Cunningham JT, Knight WD, Mifflin SW, Nestler EJ. An Essential role for DeltaFosB in the median preoptic nucleus in the sustained hypertensive effects of chronic intermittent hypoxia. Hypertension. 2012;60(1):179-187.

31. Zubcevic J, Waki H, Raizada MK, Paton JF. Auto- nomic-immune-vascular interaction: an emerging concept for neurogenic hypertension. Hypertension. 2011;57(6):1026-1033.

30. Shi $\mathrm{P}$, et al. Brain microglial cytokines in neurogenic hypertension. Hypertension. 2010;56(2):297-303.

32. Swirski FK, Nahrendorf M. Leukocyte behavior in atherosclerosis, myocardial infarction, and heart failure. Science. 2013;339(6116):161-166.

33. Guzik TJ, et al. Role of the T cell in the genesis of angiotensin II induced hypertension and vascular dysfunction. J Exp Med. 2007;204(10):2449-2460.

34. Abboud FM, Harwani SC, Chapleau MW. Autonomic neural regulation of the immune system: implications for hypertension and cardiovascular disease. Hypertension. 2012;59(4):755-762.

35. Harwani S, Chapleau MW, Legge KL, Ballas ZK, Abboud FM. Neurohormonal modulation of the innate immune response is pro-inflammatory in the pre-hypertensive spontaneously hypertensive rat. A genetic model of essential hypertension. Circ Res. 2012;111(9):1190-1197.

36. Tracey KJ. The inflammatory reflex. Nature. 2002; 420(6917):853-859.

37. Li M, Zheng C, Sato T, Kawada T, Sugimachi M, Sunagawa K. Vagal nerve stimulation markedly improves long-term survival after chronic heart failure in rats. Circulation. 2004;109(1):120-124.

38. Fogel RB, et al. Within breath control of genioglossal muscle activation in humans: Effect of sleep wake state. J Physiol. 2003;550(pt 3):899-910.

39. Basner RC. Continuous positive airway pressure for obstructive sleep apnea. $N$ Engl J Med. 2007; 356(17):1751-1758

40. Strollo PJ Jr, et al. Upper-airway stimulation for obstructive sleep apnea. $N$ Engl J Med. 2014; 370(2):139-149. 